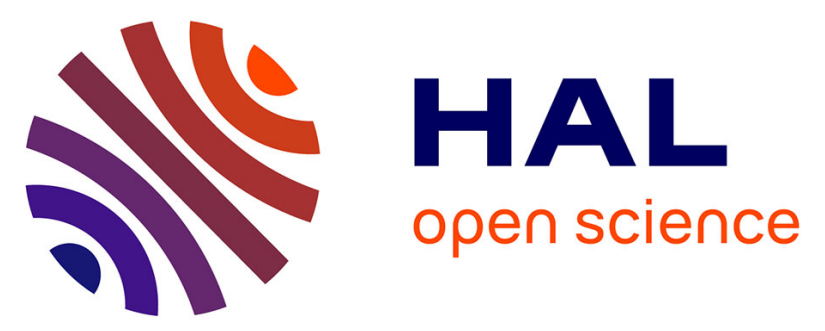

\title{
Time-frequency Selection in Two Bipolar Channels for Improving the Classification of Motor Imagery EEG
}

Yuan Yang, Sylvain Chevallier, Joe Wiart, Isabelle Bloch

\section{To cite this version:}

Yuan Yang, Sylvain Chevallier, Joe Wiart, Isabelle Bloch. Time-frequency Selection in Two Bipolar Channels for Improving the Classification of Motor Imagery EEG. The proceeding of 34th Annual International Conference of the IEEE Engineering in Medicine and Biology Society (EMBC'12), Aug 2012, San Diego, United States. pp.2744-2747. hal-00737280

\section{HAL Id: hal-00737280 \\ https://hal.science/hal-00737280}

Submitted on 1 Oct 2012

HAL is a multi-disciplinary open access archive for the deposit and dissemination of scientific research documents, whether they are published or not. The documents may come from teaching and research institutions in France or abroad, or from public or private research centers.
L'archive ouverte pluridisciplinaire HAL, est destinée au dépôt et à la diffusion de documents scientifiques de niveau recherche, publiés ou non, émanant des établissements d'enseignement et de recherche français ou étrangers, des laboratoires publics ou privés. 


\title{
Time-frequency Selection in Two Bipolar Channels for Improving the Classification of Motor Imagery EEG*
}

\author{
Yuan Yang ${ }^{1}$, Sylvain Chevallier ${ }^{2}$, Joe Wiart ${ }^{3}$, Isabelle Bloch ${ }^{1}$
}

\begin{abstract}
Time and frequency information is essential to feature extraction in a motor imagery BCI, in particular for systems based on a few channels. In this paper, we propose a novel time-frequency selection method based on a criterion called Time-frequency Discrimination Factor $(T F D F)$ to extract discriminative event-related desynchronization (ERD) features for BCI data classification. Compared to existing methods, the proposed approach generates better classification performances (mean kappa coefficient $=\mathbf{0 . 6 2}$ ) on experimental data from the BCI competition IV dataset IIb, with only two bipolar channels.
\end{abstract}

\section{INTRODUCTION}

Brain-computer interface (BCI) systems capture subject's intentions by measuring his brain activities and then translate them into commands to build a direct communication between brain and computer. Usually, brain activities are measured through electroencephalogram (EEG) because of its low cost and high time resolution [1]. One of the most typical brain activities that can be captured by EEG recording is the band power modulation of sensorimotor rhythms, i.e. $\mu$ and $\beta$ rhythms, during motor imagery [2]. Existing studies show that imaginary movements of different body parts can cause the attenuation of sensorimotor rhythms, termed as eventrelated desynchronization (ERD), at corresponding "active" cortex areas; meanwhile, an enhancement of sensorimotor rhythms called event-related synchronization (ERS) might be observed at other "idling" areas [3]. Thus, motor imagery can be identified by classifying ERD/ERS patterns, which gives birth to motor imagery BCI [1]. However, the poor signalto-noise ratio (SNR) of raw EEG signals and the overlap of different rhythms (e.g. $\alpha$ and $\mu$ rhythms) make it difficult to extract discriminative features for BCI classification [4]. Although multi-channel recording with a large number of electrodes (e.g. 64 or 128) and spatial filters can improve the SNR and extract discriminative features from overlapping signals [5], this setting reduces the portability and practicability of BCI so that it represents a main drawback for final users.

To solve this problem, several researchers proposed to record motor imagery EEG data with only a few bipolar electrodes placed around task-relevant sensorimotor areas of

\footnotetext{
*This work was partially supported by grants from China Scholarship Council and Orange Labs.

${ }^{1}$ Y. Yang and I. Bloch are with Télécom ParisTech, CNRS LTCI, and WHIST Lab, Paris, France. yuan.yang at telecom-paristech.fr, isabelle.bloch at telecom-paristech.fr

${ }^{2}$ S. Chevallier is with LISV - IUT de Vélizy, Université de Versailles StQuentin, Vélizy, France. sylvain.chevallier at uvsq.fr

${ }^{3} \mathrm{~J}$. Wiart is with Orange Labs R\&D and WHIST Lab, Issy les Moulineaux, France. joe.wiart at orange-ftgroup.com
}

the brain [6], [7]. Bipolar recording is an EEG acquisition technique that measures potential differences between two electrodes. This can improve the SNR by eliminating common additive noise of both electrodes (for details, see [6]). The positions of bipolar electrodes can be optimized algorithmically or using prior knowledge on the spatial location of brain activity during motor imagery (e.g. imagination of right hand movement elicits ERD around left sensorimotor areas). This allows reducing the number of electrodes while improving classification performances.

Beside spatial information, time and frequency information is also very important for classification, because motor imagery elicits ERD/ERS in specific bands within specific time segments instead of over all frequency bands in the whole trial. In general, $\mu$ rhythm is in $8-12 \mathrm{~Hz}$ band and $\beta$ rhythm in $18-25 \mathrm{~Hz}$ band, but these rhythms can vary with subjects. Thus, a number of algorithms were proposed to find subject-specific frequency bands for extracting discriminative features [8], [9], [10], [11], [12]. Most of them are based on extensions of the common spatial pattern (CSP) algorithm, such as filter bank CSP (FBCSP) [8], [9], subband CSP (SBCSP) [11] and common spatio-spectral pattern (CSSP) [10], which combine flexible frequency filtering techniques with CSP to improve BCI performances. Among those methods, FBCSP seems to be the most effective one, because it yields the best $\mathrm{BCI}$ performances on multiple $\mathrm{BCI}$ competition data [8], [9]. Moreover, the robust FBCSP (RFBCSP), which includes robust Minimum Covariance Determinant (MCD) estimator in the algorithm, can be applied to motor imagery BCI with only three bipolar channels. However, RFBCSP involves mutual information (MI)-based feature selection and MCD estimator, both of which needing tedious iterative steps that greatly increase its complexity. Moreover, this method used a fixed time segment (i.e. 0.5-2.5s after cue on-set) of the EEG data for all subjects in the training process, which ignores the potential individual difference in optimal time segment selection.Although a recent work on FBCSP has involved optimal temporal selection process [13], it selected the time segment from only four different options $(0.5-2.5 \mathrm{~s}, 1.0-3.0 \mathrm{~s}, 1.5-3.5 \mathrm{~s}$ and $0.5-3.5 \mathrm{~s})$ and did not led to significant improvement on bipolar channel data (BCI competition IV IIb) compared to previous results in [8].

In this paper, we propose a time-frequency selection method for extracting subject-specific ERD features for further improving motor imagery EEG classification with only a few bipolar electrodes. This method is based on a proposed criterion called Time-frequency Discrimination Factor (TFDF) to extract discriminative ERD features for the 
classification. Compared with the methods mentioned above, the proposed method is different in the following ways: 1) this method is neither based on CSP algorithm nor combined with other complex algorithms (e.g. MI-based algorithms or MCD estimator); 2) the subject-specific time-frequency area is selected from more than one thousand possible areas by a novel time-frequency selection criteria (TFDF), which can further improve the classification performances on bipolar recording BCI data; 3) this method can be applied on data recorded with only two bipolar channels for binary-class problems (e.g. a discrimination between imaginations of left and right hand movements).

\section{EXPERIMENTAL DATA}

We used data of the BCI competition IV dataset IIb [7] in this work. The dataset consists of two classes (left vs. right hand) cue-driven motor imagery BCI data from 9 subjects. The EEG data are recorded using 3 bipolar channels, i.e. at positions $\mathrm{C} 3, \mathrm{Cz}$ and $\mathrm{C} 4$. For each subject, 5 sessions are provided, including 3 training sessions and 2 evaluation sessions. The first two training sessions consist of 240 single trials (120 trials per session) without visual feedback. Each trial starts with a fixation cross and a short acoustic warning tone. Later a visual cue is given to guide the subject to execute the corresponding imagination of hand movement over a period of 4 seconds. The last training session (160 trials) and the two evaluation sessions (160 trials per session) are recorded with visual feedback from 0.5 to $4.5 \mathrm{~s}$ after the cue on-set (for details, see [7]).

Many studies have shown that the electrode positions approximately overlaying the hand representation areas i.e. C3 (right hand) and C4 (left hand), usually generate the best results of single-trial discrimination between imaginations of left and right hand movements in non-CSP based classification [3], [14], while $\mathrm{Cz}$ located at the functional area related to foot movement mainly contributes to the best discrimination between tasks involving imagination of foot movement [3]. Thus, we only used C3 and C4 in this study for improving the left v.s. right hand classification by using the proposed method.

\section{TIME-FREQUENCY ANALYSIS AND SELECTION}

\section{A. Visualization of ERD/ERS in the Time-frequency Domain}

The ERD/ERS patterns are usually expressed as percentage power decrease (ERD) or power increase (ERS) referring to the 1-s interval before the warning tone (for details, see [3]). The time-frequency maps of ERD/ERS for both left $(L)$ and right $(R)$ hands in the bipolar channels $\mathrm{C} 3$ and $\mathrm{C} 4$ were generated by the Biosig Toolbox using overlapping $2 \mathrm{~Hz}$ bands $(\mathrm{step}=1 \mathrm{~Hz}$ ) in the frequency range between 6 and $32 \mathrm{~Hz}$ [15]. The obtained time-frequency maps are used to show the significant ERD/ERS ( $t$-percentile bootstrap, $\alpha=0.05$ ) during the imaginations of right and left hand movements in $\mathrm{C} 3$ and $\mathrm{C} 4$, and to validate the necessity and effectiveness of the proposed time-frequency selection method in feature extraction (see Section IV).

\section{B. Subject-specific Time-frequency Selection}

Firstly, the signals in both channels are divided into EEG segments, in a series of overlapping time-frequency areas $\left\{\left(\omega_{m}, \tau_{n}\right)\right\}_{M \times N}$ with different frequency bands $\omega_{m}=$ $\left[f_{m}, f_{m}+F-1\right], m=1, \ldots, M, f_{m+1}=f_{m}+F_{s}(F$ is the bandwidth, $F_{S}$ is the frequency step) and time intervals $\tau_{n}=$ $\left[t_{n}, t_{n}+T-1\right], n=1, \ldots, N, t_{m+1}=t_{m}+T_{s}$ ( $T$ is the interval width, $T_{s}$ is the time step).

As ERD features for the BCI classification are often measured by the logarithm of variance of band-pass filtered EEG in a specific time interval, so called logarithmic band power $(B P)$ estimator [16], the overall $B P$ of EEG segment ( $K$ trials) in a time-frequency area $\left(\omega_{m}, \tau_{n}\right)$ for each condition and each channel is estimated as follows.

Step 1: The variance of EEG segment in the time domain for each trial $i, i=1, \ldots, K$ for each condition and each channel is computed as:

$$
v(i)=\frac{1}{T-1} \sum_{j=t_{n}}^{t_{n}+T-1}\left(x_{i j}-\bar{x}_{i}\right)^{2}
$$

where $x_{i j}$ the $j$-th sample in the time interval $\tau_{n}$ of the $i$-th trial of the $\omega_{m}$-bandpass filtered EEG data, and $\bar{x}_{i}$ is the mean value over all samples of filtered EEG in the time interval $\tau_{n}$ of the $i$-th trial.

Step 2: According to the existing literature, the overall $B P$ for each class $(L, R)$ and each channel $(\mathrm{C} 3, \mathrm{C} 4)$ is calculated as the logarithm of median or mean of data variances over trials. Here, we use medians instead of mean values in the calculation, because outliers in the experimental data we used were reported, e.g. in [8].

$$
\begin{aligned}
& B P_{C 3}^{L}=\log \left(\operatorname{med}\left(v_{C 3}^{L}(i)\right)\right) \\
& B P_{C 4}^{L}=\log \left(\operatorname{med}\left(v_{C 4}^{L}(i)\right)\right) \\
& B P_{C 3}^{R}=\log \left(\operatorname{med}\left(v_{C 3}^{R}(i)\right)\right) \\
& B P_{C 4}^{R}=\log \left(\operatorname{med}\left(v_{C 4}^{R}(i)\right)\right)
\end{aligned}
$$

where $\operatorname{med}(\cdot)$, denotes the median of the data over all trails, $i=1, \ldots, K$, for each condition and each channel.

Thus, the pattern difference $(P D)$ between two conditions (left vs. right hand) in time-frequency area $\left(\omega_{m}, \tau_{n}\right)$ at each channel is expressed as:

$$
\begin{aligned}
& P D_{C 3}=B P_{C 3}^{L}-B P_{C 3}^{R} \\
& P D_{C 4}=B P_{C 4}^{L}-B P_{C 4}^{R}
\end{aligned}
$$

Imaginations of left and right hand movement usually elicited contrary contralateral dominance of ERD at C3 and C4 [3], [14]. These task-spatial-related discriminative modulations can be measured by discriminative force $F d\left(\omega_{m}, \tau_{n}\right)$ to estimate this positive contribution in a time-frequency area $\left(\omega_{m}, \tau_{n}\right)$ for the classification:

$$
\begin{aligned}
F d\left(\omega_{m}, \tau_{n}\right) & =\left|P D_{C 3}-P D_{C 4}\right| \\
& =\left|\log \left(\frac{\operatorname{med}\left(v_{C 3}^{L}(i)\right)}{\operatorname{med}\left(v_{C 3}^{R}(i)\right)} / \frac{\operatorname{med}\left(v_{C 4}^{L}(i)\right)}{\operatorname{med}\left(v_{C 4}^{R}(i)\right)}\right)\right|
\end{aligned}
$$


A large $F d\left(\omega_{m}, \tau_{n}\right)$ indicates that large discriminative modulations occur in the time-frequency area $\left(\omega_{m}, \tau_{n}\right)$.

On the other hand, subjects are looking at the screen during both motor imagery tasks, which can generate some nonmotor-imagery-related (i.e. visual related) common modulations at $\mathrm{C} 3$ and $\mathrm{C} 4$ [5]. Meanwhile, neural activities at $\mathrm{C} 3$ and $\mathrm{C} 4$ may also affect each other due to volume conduction effects. These possible negative effects in the time-frequency area $\left(\omega_{m}, \tau_{n}\right)$ on the classification are estimated using a blurring force $F b\left(\omega_{m}, \tau_{n}\right)$ :

$$
\begin{aligned}
F b\left(\omega_{m}, \tau_{n}\right) & =\left|P D_{C 3}+P D_{C 4}\right| \\
& =\left|\log \left(\frac{\operatorname{med}\left(v_{C 3}^{L}(i)\right)}{\operatorname{med}\left(v_{C 3}^{R}(i)\right)} \cdot \frac{\operatorname{med}\left(v_{C 4}^{L}(i)\right)}{\operatorname{med}\left(v_{C 4}^{R}(i)\right)}\right)\right|
\end{aligned}
$$

A small $F b\left(\omega_{m}, \tau_{n}\right)$ indicates that small common modulations happen in the time-frequency area $\left(\omega_{m}, \tau_{n}\right)$.

We call the difference between $F d\left(\omega_{m}, \tau_{n}\right)$ and $F b\left(\omega_{m}, \tau_{n}\right)$ Time-frequency Discrimination Factor, TFDF $\left(\omega_{m}, \tau_{n}\right)$, and use it to evaluate the overall contribution of the data in the time-frequency area $\left(\omega_{m}, \tau_{n}\right)$ from electrodes $\mathrm{C} 3$ and $\mathrm{C} 4$ for the binary-class discrimination:

$$
\operatorname{TFDF}\left(\omega_{m}, \tau_{n}\right)=F d\left(\omega_{m}, \tau_{n}\right)-F b\left(\omega_{m}, \tau_{n}\right)
$$

An ideal time-frequency area for classification should have large discriminative modulations and small common modulations, so that the optimal time-frequency area $\left(\omega_{\text {opt }}, \tau_{\text {opt }}\right)$ is estimated by seeking the maximum value of $\operatorname{TFDF}\left(\omega_{m}, \tau_{n}\right)$ among all time-frequency areas.

\section{Data Elaboration, Feature Extraction and Classification}

In this study, the sessions used for training are selected based on the suggestions of winners in BCI competition IV [17] in order to compare our results with theirs. For each bipolar channel, 5th order butterworth filters are applied to compute 19 successive $4 \mathrm{~Hz}$-wide frequency bands of signals: $8-12 \mathrm{~Hz}, 9-13 \mathrm{~Hz}, 10-14 \mathrm{~Hz}, \ldots, 26-30 \mathrm{~Hz}$, and 15 successive $8 \mathrm{~Hz}$-wide frequency bands of signals: $8-16 \mathrm{~Hz}, 9-17 \mathrm{~Hz}, 10-$ $18 \mathrm{~Hz}, \ldots, 22-30 \mathrm{~Hz}(M=34)$. Then 39 overlapping time segments in each frequency band were obtained through $2 \mathrm{~s}$, $2.5 \mathrm{~s}$ and $3 \mathrm{~s}$-wide sliding windows (13 segments for each sliding window), respectively, with $0.2 \mathrm{~s}$-step moving from $0.5 \mathrm{~s}$ after the cue on-set $(N=39)$. Those parameters are set based on the experience from competitors reported in BCI competition IV [17]. Therefore, there are $34 \times 39=1326$ time-frequency areas for subject-specific selection. The $B P$ values are extracted from two channels of EEG in the selected time-frequency area $\left(\omega_{\text {opt }}, \tau_{\text {opt }}\right)$ as ERD features. Then, Fisher's linear discriminant analysis (LDA) was employed with these features for the classification as most BCI classification works did [18].

\section{EXPERIMENTAL RESULTS AND DISCUSSION}

Fig. 1 shows an example of time-frequency maps displaying significant ERD (red) and ERS (blue) for a typical subject (Subject 6) in BCI competition IV dataset IIb [7]. From Fig. 1, we can see that 1) imagination of left hand movement elicits $\mathrm{C} 4$ dominant $\mu$ band ERD patterns while that of right hand movement generates $\mathrm{C} 3$ dominant $\mu$ band ERD patterns around $12 \mathrm{~Hz}$ approximately during $0.5-5 \mathrm{~s}$ after cue on-set; 2) a background noise (generated by non-motorimagery-related activities) is widely observed in the timefrequency map (e.g. in $15-30 \mathrm{~Hz}, 1-4 \mathrm{~s}$ ) so that information from those areas may deteriorate the classification results. Based on these observations, we can conclude that 1) timefrequency selection is necessary for extracting discriminative ERD patterns for a good classification performance; 2) common noise cannot be completely removed in all timefrequency areas through bipolar recoding technique. Thus, a subject-specific time-frequency selection method is required to capture the ERD features in the two bipolar channels that contain discriminative information.

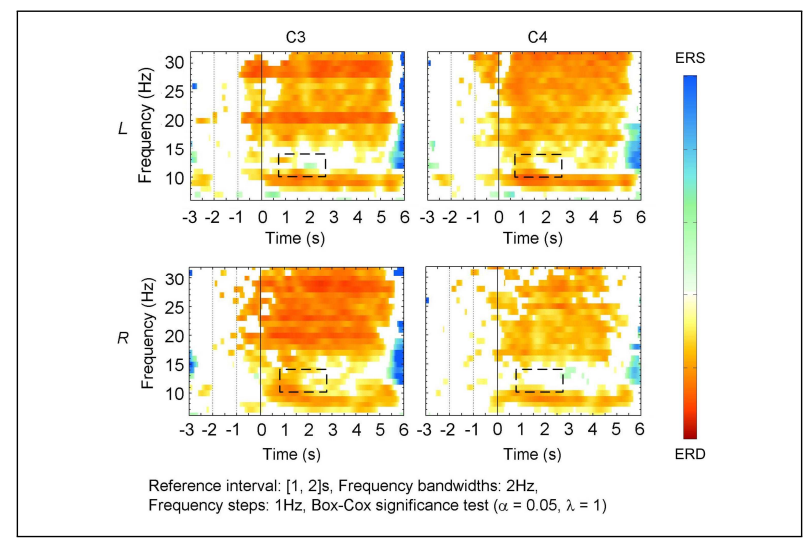

Fig. 1. Time-frequency maps displaying significant ERD (red) and ERS (blue) for Subject 6 (a typical example) in BCI competition IV IIb. The areas in the rectangles are the time-frequency areas selected by the proposed method. Imaginations of left and right hand movement elicited contrary contralateral dominant ERD in the selected areas, which indicates the effectiveness of the proposed method in time-frequency selection for discrimination.

Table I lists the optimal frequency band $\omega_{\text {opt }}$ and time interval $\tau_{\text {opt }}$ of the time-frequency areas selected from the training data by the proposed method for each subject. For example, the optimal frequency band of Subject 6 is 10$14 \mathrm{~Hz}$ and the optimal time segment is $0.7-2.7 \mathrm{~s}$, which is slightly different from the general $\mu$ band $(8-12 \mathrm{~Hz})$ and the suggested time segment $(0.5-2.5 \mathrm{~s})$ in the literature [8]. From Fig. 1, we can see that this time-frequency area contains the discriminate ERD patterns of two classes: imaginations of left hand and right hand movements elicited contrary contralateral ERD dominance in the selected areas, which indicates the effectiveness of the proposed method in timefrequency selection for discrimination. The optimal time segments and frequency bands for capturing discriminate ERD patterns vary with different subjects (see Table I), so that it is necessary to use subject-specific time-frequency area in the classification.

In the classification, the EEG signals in the subjectspecific time-frequency area $\left(\omega_{\text {opt }}, \tau_{\text {opt }}\right)$ from all training trials are used for training, then the $\omega_{o p t}$-bandpass filtered EEGs segments with the same time length of $\tau_{\text {opt }}$ (i.e. $T$ ) are obtained from each entire single-trial of testing data via a 
TABLE I

FREQUENCY BAND $\omega_{o p t}$ AND TIME INTERVAL $\tau_{o p t}$ Selected By the TFDF VAlues For All the Subjects.

\begin{tabular}{|c|c|c|c|c|c|c|c|c|c|}
\hline & \multicolumn{10}{|c|}{ Subjects } \\
\cline { 2 - 8 } & 1 & 2 & 3 & 4 & 5 & 6 & 7 & 8 & 9 \\
\hline$\omega_{\text {opt }}(H z)$ & $10-14$ & $11-15$ & $9-13$ & $8-12$ & $26-30$ & $10-14$ & $11-15$ & $10-14$ & $22-26$ \\
$\tau_{\text {opt }}(s)$ & $2.9-4.9$ & $2.7-5.2$ & $0.5-2.5$ & $0.9-2.9$ & $0.7-3.2$ & $0.7-2.7$ & $1.1-3.1$ & $0.9-2.9$ & $0.9-2.9$ \\
\hline
\end{tabular}

0.2s step sliding window to generate continuous classification outputs. According to the BCI competition requirement, the classification performance is measured by kappa coefficient $\kappa$ value [19]. Table II provides the mean performance over the two independent evaluation sessions obtained by our method, and comparisons with the three best submissions of the BCI competition IV on this dataset [17]. Our method generates the best mean kappa value $(\bar{\kappa}=0.62)$ among all methods on these data. To further analyze the results, the paired-sample $t$-test was employed to reveal the statistically significant difference between the performances of different methods. Although improvements of kappa values compared to $1^{\text {st }}$ winner $(\bar{\kappa}=0.60, p=0.12)$, who used RFBCSP [8] and $2^{\text {nd }}$ winner $(\bar{\kappa}=0.58, p=0.18)$, who applied common spatial subspace decomposition (CSSD) on multiply frequency bands [17], are not statistically significant, our method outperforms the $1^{\text {st }}$ winner in 6 out of 9 subjects (except subjects 4, 7 and 8 ), and the $2^{\text {nd }}$ winner in 6 out of 9 subjects (except subjects 4, 7 and 9). For this dataset, our results are always better than the $3^{\text {rd }}$ placed one $(\bar{\kappa}=$ $0.46, p<0.01$ ), who employed classical CSP with spectrally filtered neural time series prediction preprocessing (NTSPP) signals [17]. As all of their works have used all three bipolar channels $(\mathrm{C} 3, \mathrm{Cz}$ and $\mathrm{C} 4)$ provided by the dataset, our method not only generated better mean performances but also used less channels than them, which shows the interest of our method in feature extraction and electrode reduction.

TABLE II

Comparison of the Classification Performances between the Proposed TFDF Method And Other Methods.

\begin{tabular}{|c|c|c|c|c|c|c|c|c|c|c|}
\hline & \multicolumn{10}{|c|}{ Subjects } \\
\cline { 2 - 10 } & 1 & 2 & 3 & 4 & 5 & 6 & 7 & 8 & 9 & Mean \\
\hline TFDF & 0.44 & 0.24 & 0.25 & 0.93 & 0.86 & 0.70 & 0.55 & 0.85 & 0.75 & 0.62 \\
\hline RFBCSP $\left(1^{\text {st }}\right)$ & 0.40 & 0.21 & 0.22 & 0.95 & 0.86 & 0.61 & 0.56 & 0.85 & 0.74 & 0.60 \\
CSSD $\left(2^{\text {nd }}\right)$ & 0.42 & 0.21 & 0.14 & 0.94 & 0.71 & 0.62 & 0.61 & 0.84 & 0.78 & 0.58 \\
CSP+NTSPP $\left(3^{r d}\right)$ & 0.19 & 0.12 & 0.12 & 0.77 & 0.57 & 0.49 & 0.38 & 0.85 & 0.61 & 0.46 \\
\hline
\end{tabular}

\section{CONCLUSION}

Previous state of the art has highlighted the need for user-specific parametrization in the preprocessing steps of motor imagery BCI, especially in term of frequency band adaptation. This contribution, through the proposition of a novel algorithm and a new criterion, emphasizes that the calibration in the temporal domain is also relevant. The results show that our approach yields better average performance using only two bipolar channels and a simple LDA classifier compared to those obtained by existing methods on the BCI competition dataset IIb. However, this conclusion has to be confirmed on more datasets; and a deeper study of the differences with respect to existing methods should be conducted. In the future, we will also extend this method to multi-class problems.

\section{REFERENCES}

[1] J. Wolpaw, N. Birbaumer, D. McFarland, G. Pfurtscheller, and T. Vaughan, "Brain-computer interfaces for communication and control," Clin. Neurophysiol., vol. 113, no. 6, pp. 767-791, 2002.

[2] G. Pfurtscheller and F. Lopes da Silva, "Event-related EEG/MEG synchronization and desynchronization: basic principles," Clin. Neurophysiol., vol. 110, no. 11, pp. 1842-1857, 1999.

[3] G. Pfurtscheller, C. Brunner, A. Schlogl, and F. Lopes da Silva, "Mu rhythm (de)synchronization and EEG single-trial classification of different motor imagery tasks," NeuroImage, vol. 31, no. 1, pp. 153-159, 2006.

[4] M. Naeem, C. Brunner, and G. Pfurtscheller, "Dimensionality reduction and channel selection of motor imagery electroencephalographic data," Comput. Intell. Neurosci., vol. 2009, pp. 1-8, 2009.

[5] B. Blankertz, R. Tomioka, S. Lemm, M. Kawanabe, and K. Müller, "Optimizing spatial filters for robust EEG single-trial analysis," IEEE Signal Process. Mag., vol. 25, no. 1, pp. 41-56, 2008.

[6] B. Lou, B. Hong, X. Gao, and S. Gao, "Bipolar electrode selection for a motor imagery based brain-computer interface," J Neural Eng., vol. 5, pp. 342-349, 2008.

[7] R. Leeb, F. Lee, C. Keinrath, R. Scherer, H. Bischof, and G. Pfurtscheller, "Brain-computer communication: motivation, aim, and impact of exploring a virtual apartment," IEEE Trans. Neural Syst. Rehabil. Eng., vol. 15, no. 4, pp. 473-482, 2007.

[8] K. Ang, Z. Chin, H. Zhang, and C. Guan, "Robust filter bank common spatial pattern (RFBCSP) in motor-imagery-based braincomputer interface," in Conf. Proc. IEEE Eng. Med. Biol. Soc. (EMBC 2009), 2009, pp. 578-581.

[9] Z. Chin, K. Ang, C. Wang, C. Guan, and H. Zhang, "Multi-class filter bank common spatial pattern for four-class motor imagery BCI," in Conf. Proc. IEEE Eng. Med. Biol. Soc. (EMBC 2009), 2009, pp. 571574.

[10] S. Lemm, B. Blankertz, G. Curio, and K. Muller, "Spatio-spectral filters for improving the classification of single trial EEG," IEEE Trans. on Biomed. Eng., vol. 52, no. 9, pp. 1541-1548, 2005.

[11] Q. Novi, C. Guan, T. Dat, and P. Xue, "Sub-band common spatial pattern (SBCSP) for brain-computer interface," in Conf. Proc. IEEE/EMBS Neural Eng. (CNE'07), 2007, pp. 204-207.

[12] N. Yamawaki, C. Wilke, Z. Liu, and B. He, "An enhanced timefrequency-spatial approach for motor imagery classification," IEEE Trans. Neural Syst. and Rehabil. Eng., vol. 14, no. 2, pp. 250-254, 2006

[13] K. Ang, Z. Chin, H. Zhang, and C. Guan, "Mutual information-based selection of optimal spatial-temporal patterns for single-trial EEGbased BCIs," Pattern Recogn., vol. 45, pp. 2137-2144, 2011.

[14] G. Pfurtscheller, C. Neuper, D. Flotzinger, and M. Pregenzer, "EEGbased discrimination between imagination of right and left hand movement," Electroencephalogr. Clin. Neurophysiol., vol. 103, no. 6 , pp. 642-651, 1997.

[15] A. Schlogl and C. Brunner, "Biosig: a free and open source software library for BCI research," Computer, vol. 41, no. 10, pp. 44-50, 2008.

[16] C. Vidaurre, N. Kramer, B. Blankertz, and A. Schlogl, "Time domain parameters as a feature for EEG-based brain-computer interfaces," Neural Netw., vol. 22, no. 9, pp. 1313-1319, 2009.

[17] B. Blankertz, "BCI competition IV," 2008, http://www.bbci.de/competition/iv/.

[18] F. Lotte, M. Congedo, A. Lécuyer, F. Lamarche, and B. Arnaldi, "A review of classification algorithms for EEG-based brain-computer interfaces," J Neural Eng., vol. 4, pp. R1-R13, 2007.

[19] G. Dornhege, Toward brain-computer interfacing. The MIT Press, 2007. 